\title{
A Classic Adamantinoma Arising from Osteofibrous Dysplasia-like Adamantinoma in the Lower Leg: A Case Report and Review of the Literature
}

\author{
Masahito Hatori, Mika Watanabe, ${ }^{1}$ Masami Hosaka, Hironobu Sasano, ${ }^{1}$ \\ Masaharu Narita ${ }^{2}$ and Shoichi KoKUbun \\ Department of Orthopaedic Surgery, ${ }^{2}$ Department of Pathology, \\ Tohoku University School of Medicine, Sendai, Japan, and \\ ${ }^{2}$ Department of Orthopaedic Surgery, Tome City Sanuma Hospital, Tome, Japan
}

Hatori, M., Watanabe, M., Hosaka, M., Sasano, H., Narita, M. and Kokubun, S. A Classic Adamantinoma Arising from Osteofibrous Dysplasia-like Adamantinoma in the Lower Leg: A Case Report and Review of the Literature. Tohoku J. Exp. Med., 2006, 209 (1), 53-59 _ Adamantinoma is known as a low-grade malignant biphasic tumor. Classic adamantinoma is in general characterized by admixture of both epithelial and osteofibrous components that are associated with various proportions and differentiation patterns. Osteofibrous dysplasia (OFD) is a self-limited benign fibro-osseous lesion of bone during infancy and childhood. OFD-like adamantinoma is characterized by predominance of osteofibrous tissues, in which small groups of epithelial cells are only detected by careful search or immunohistochemistry. There have been controversies as to the potential correlation among OFD, OFD-like adamantinoma and classic adamantinoma. We report an unusual case of adamantinoma arising in the tibia, with an extensive review of the literature. The present findings suggest a direct correlation between OFD-like adamantinoma and classic adamantinoma. At the age of 12 years, the tibial biopsy lesion was diagnosed as OFD. At the age of 23 , the lesion became larger and more destructive on $\mathrm{x}$-ray films. The biopsy lesion was diagnosed as classic adamantinoma. Wide excision was performed. The primary lesion was retrospectively diagnosed as OFD-like adamantinoma because of presence of keratin-positive epithelial cells within the stroma. At five years after surgery, the patient was free from recurrence or metastasis. The retrospective histological findings of OFD-like adamantinoma in the original biopsy and of a classic adamantinoma in all sections of the later resection specimen raised the possibility of an unusual progression of OFD-like adamantinoma to a classic adamantinoma. The present case and the literature review suggest that an OFD-like adamantinoma may be a precursor lesion of classic adamantinoma. Therefore, the possibility of progression of OFD-like adamantinoma to a classic adamantinoma should be kept in mind, particularly when the destructive changes are seen radiologically. — adamantinoma; osteofibrous dysplasia; osteofibrous dysplasialike adamantinoma; classic adamantinoma; tibia (C) 2006 Tohoku University Medical Press

Received January 10, 2006; revision accepted for publication March 3, 2006.

Correspondence: Masahito Hatori, M.D., Department of Orthopaedic Surgery, Tohoku University School of Medicine, Seiryomachi, Aoba-ku, Sendai 980-8574, Japan.

e-mail:mhato@mail.tains.tohoku.ac.jp 
Adamantinoma is a low grade, malignant biphasic tumor characterized by a variety of morphological patterns, most commonly epithelial cells, surrounded by a relatively bland spindlecell osteofibrous component (Hogendoorn and Hashimoto 2002). It comprises about $0.4 \%$ of all primary bone tumors (Huvos 1991). Classic adamantinomas are characterized by an admixture of both epithelial and osteofibrous components, which may be intermingled with each other in various proportions and differentiation pattern. Osteofibrous dysplasia (OFD) is a self-limited benign fibro-osseous lesion of bone during infancy and childhood (Vigorita et al. 2002). OFD-like adamantinoma is characterized by predominance of osteofibrous tissues, in which small groups of epithelial cells are only encountered by careful light microscopic examination or immunohistochemistry (Hogendoorn and Hashimoto 2002). In view of the still disputed potential correlation between classic adamantinoma and OFD-like adamantinoma in children, a very unique case of adamantinoma, indicating a direct relationship between the two lesions, is presented here with a review of the literature.

\section{Case Report}

At the age of 12 years, the patient noticed tenderness in her left lower leg after running. There was no past history of the trauma in that lesion. Subsequent radiological examination revealed a lesion with multiple lucencies with intervening sclerosis in the diaphysis of the left tibia and the distal metaphysis of the ipsilateral fibula. The cortex was thinned (Fig. 1). Open biopsy was performed. The lesion was diagnosed as OFD. Removal of the tumor was not performed because her pain disappeared. At the age of 20, swelling appeared in her left proximal lower leg. She visited the surgeon at the age of 23 due to an aggravation of the pain. The patient was referred to Tohoku University Hospital. An 8 $\times 8 \mathrm{~cm}$ sized elastic hard mass was palpated in the proximal part of the left lower leg. Mild local heat and tenderness were noted. Plain radiography showed progression of multiple lucencies in the tibia. The lesion was larger and more destruc-
A

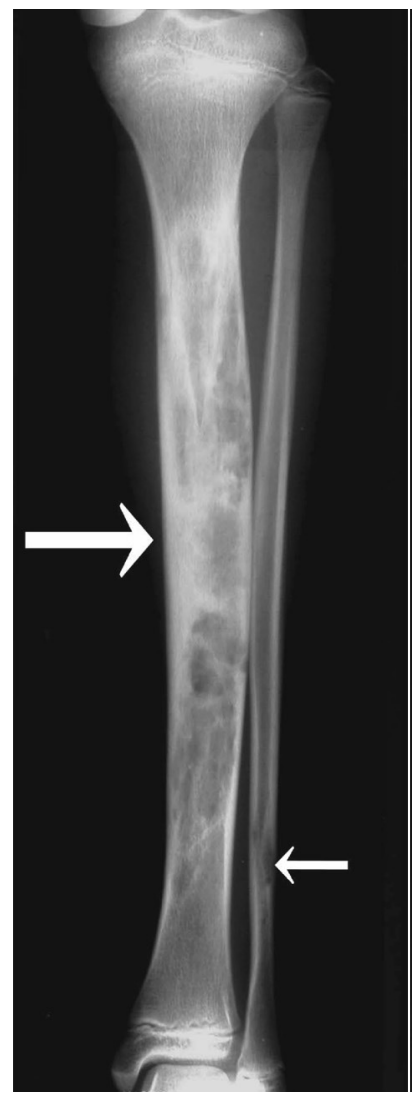

B

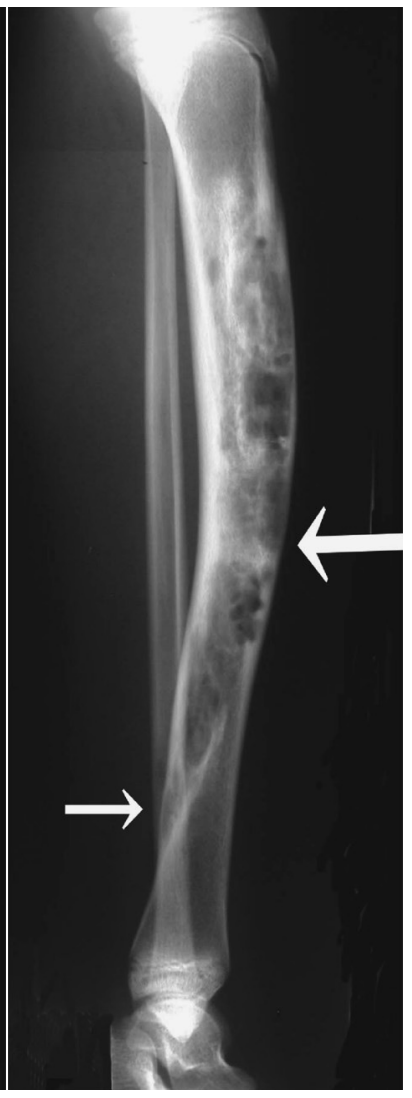

Fig. 1. Plain radiography showing lesions with multiple lucencies with intervening sclerosis with partially ground glass appearance in the diaphysis of the left tibia (large arrow) and the distal portion of the ipsilateral fibula (small arrow). The cortex was thinned (A: anteroposterior view, B: lateral view).

tive as compared with the plain radiogram at the age of 12. The cortex was expanded and thinned and partially disrupted with multiple radiolucencies mixed with intervening areas of sclerosis (Fig. 2). Computed tomography demonstrated extraskeletal growth of the lesion with cortical destruction (Fig. 3). Magnetic resonance imaging clearly visualized a tumor with extraskeletal growth with iso signal intensities on T1 weighted images (Fig. 4) and high signal intensities on T2 weighted images. The mass was enhanced after gadolinium injection on T1 weighted images. Open biopsy was performed again to confirm the diagnosis. Macroscopically, the lesion was com- 
A

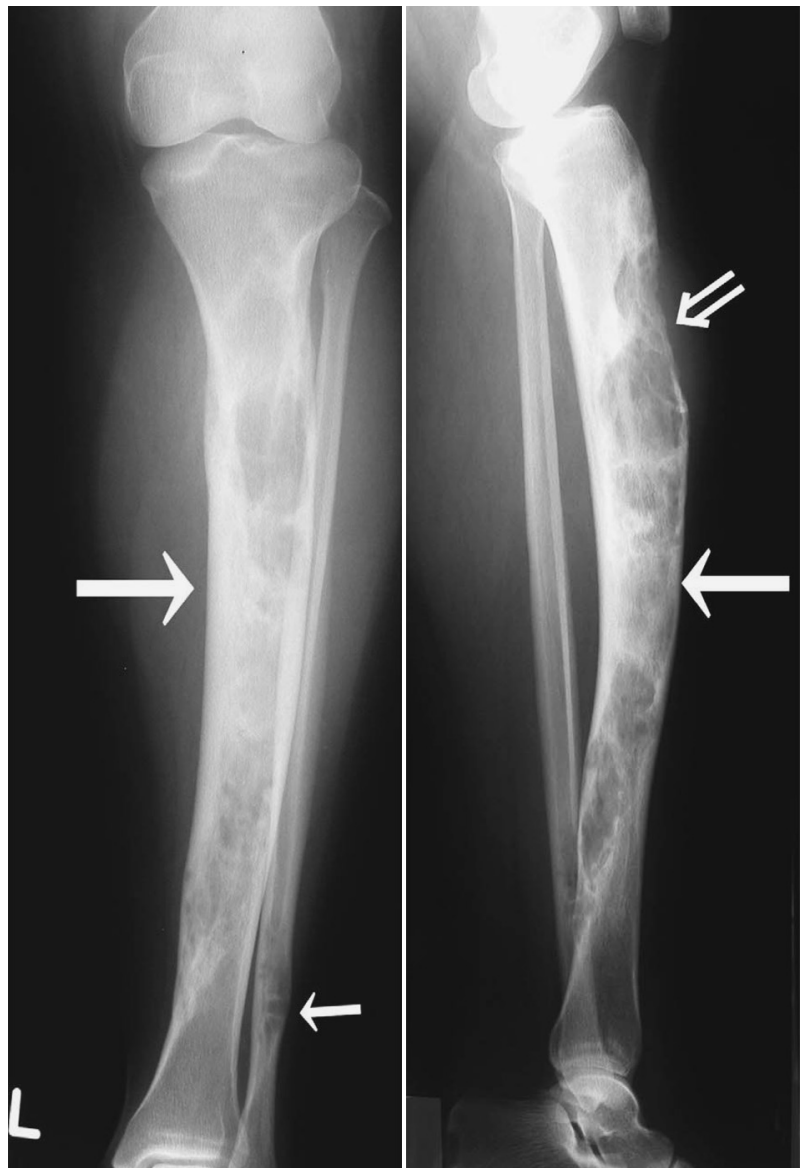

Fig. 2. Plain radiography showing the cortex was expanded and thinned and partially disrupted $(\Rightarrow)$ with multiple radiolucencies mixed with intervening areas of sclerosis.

A: anteroposterior view, B: lateral view.

Large arrow, tibial lesion; small arrow, fibula lesion.

prised of soft granular tissue which appeared whitish yellow in color. Microscopically, the tumor was composed of prominent epithelial islands admixed with intervening stroma. Spindle cell patterns suggesting fibrosarcoma were partially detected. No OFD patterns were detected (Fig. 5). The tumor cells were positive for AE1/ AE3 (pancytokeratin) $(1: 300$, DAKO, Glostrup, Denmark)(Fig. 6). The tumor was subsequently diagnosed as classic adamantinoma. Retrospective histological examination of the primary biopsy specimen taken at the age of 12 revealed that the lesion was composed of a

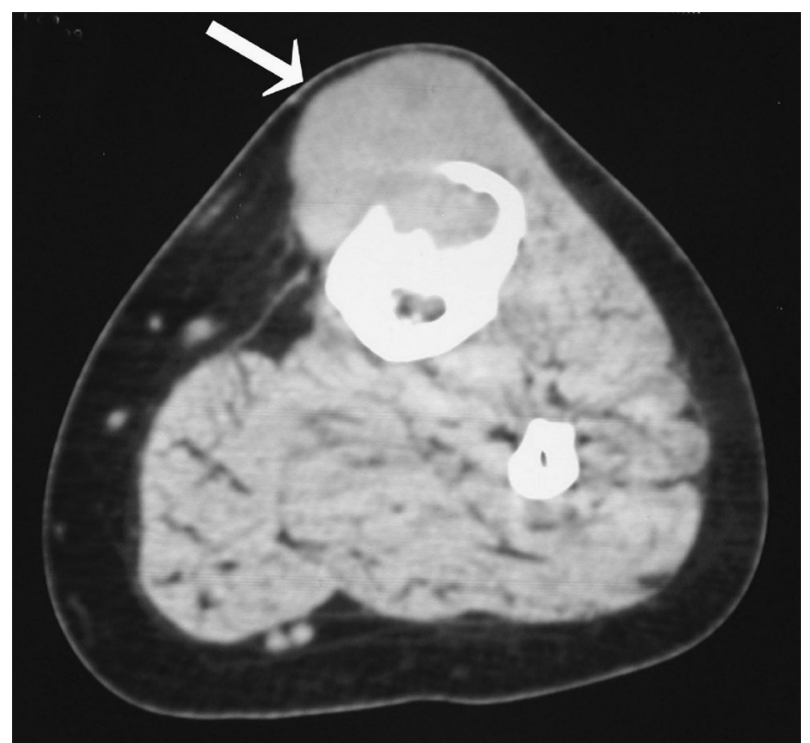

Fig. 3. Computed tomography demonstrating extraskeletal growth of the lesion (arrow) with cortical destruction.

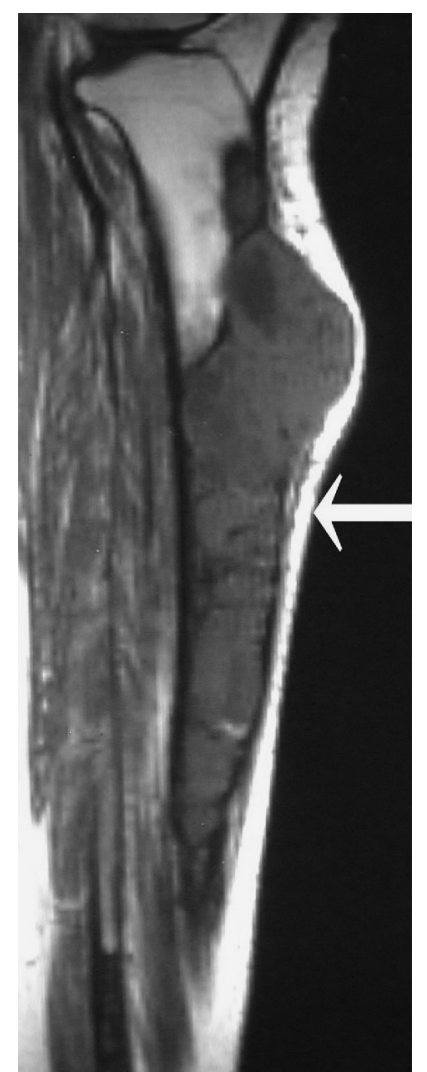

Fig. 4. Magnetic resonance imaging clearly visualizing a tumor (arrow) with extraskeletal growth with iso signal intensities on $\mathrm{T} 1$ weighted images. 
A

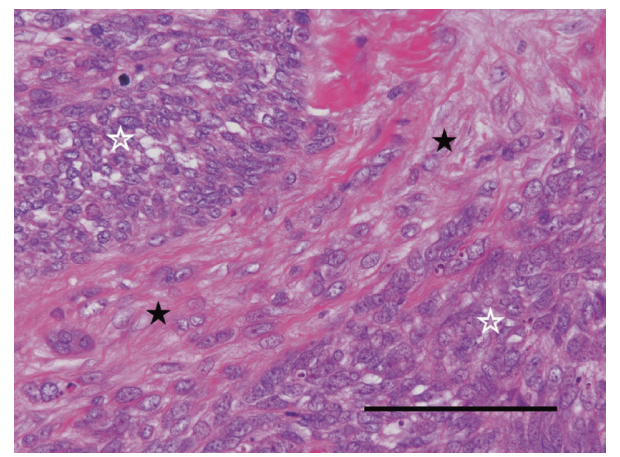

B

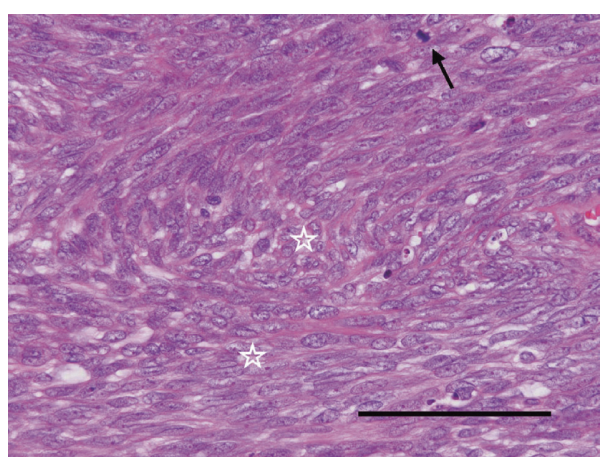

Fig. 5. Microphotographs of the biopsy specimen at the age of 23. The tumor was composed of prominent epithelial islands $(\hbar)$ with intervening stroma $(\star)(\mathrm{A})$ and spindle cell proliferation with interlacing fascicles (㧒). Mitosis was seen (arrow) (B) (Scale $100 \mu)$.

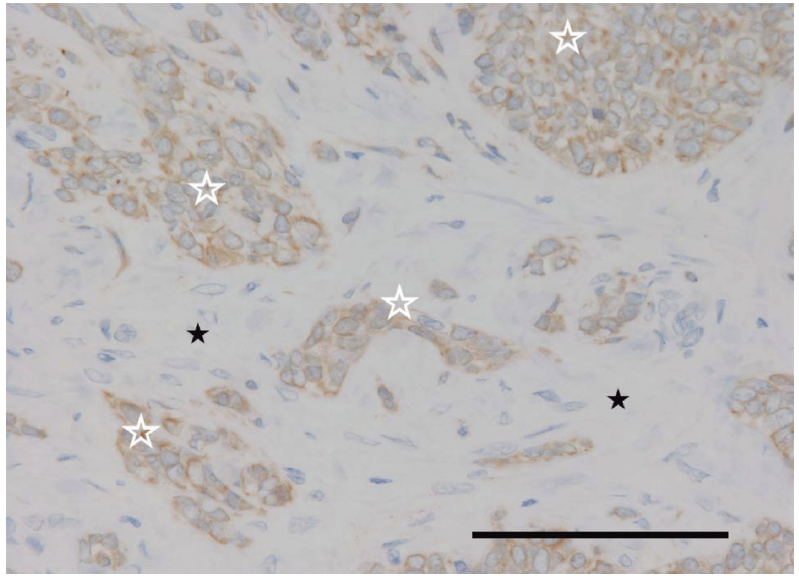

Fig. 6. Microphotograph showing that the tumor cells $(\lesssim)$ were positive for AE1/AE3 (pancytokeratin). The intervening stromal cells were not reactive for AE1/AE3. (Scale $100 \mu$ ). bland fibrous tissue, embedded small trabeculae of woven bones rimmed by prominent cubic osteoblasts. The fibrous component had delicate or coarse collagen fibers (Fig. 7). Immunohistochemical examination performed at this time demonstrated isolated keratin-positive epithelial cells within the stroma (Fig. 8). The lesion at the age of 12 was therefore retrospectively diagnosed as OFD-like adamantinoma.

Wide excision by using endoprostheis and ipsilateral vascularized fibula transfer was performed as a curative surgery. Postoperative histological examination demonstrated classic adamantinoma. No OFD like pattern was detected. At five years after surgery, the patient was free from recurrence or pulmonary metastasis.
A

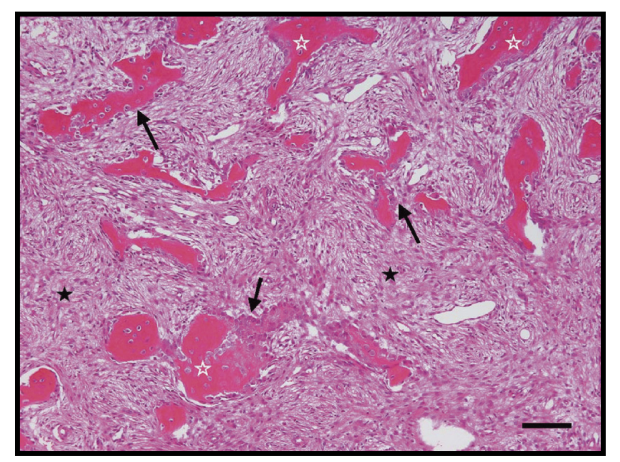

B

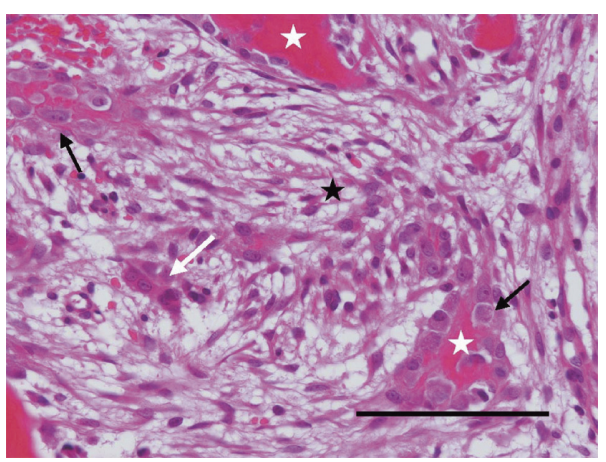

Fig. 7. Microphotograph at the age of 13, showing that the lesion was composed of a bland fibrous tissue $(\star)$, embedded small trabeculae of woven bones $(\tau)$ rimmed by prominent cubic osteoblasts (black arrow). Small epithelial cluster (white arrow) was seen in the fibrous stroma. (A: $\times 10, \mathrm{~B}: \times 40)$ (Scale $100 \mu$ ). 


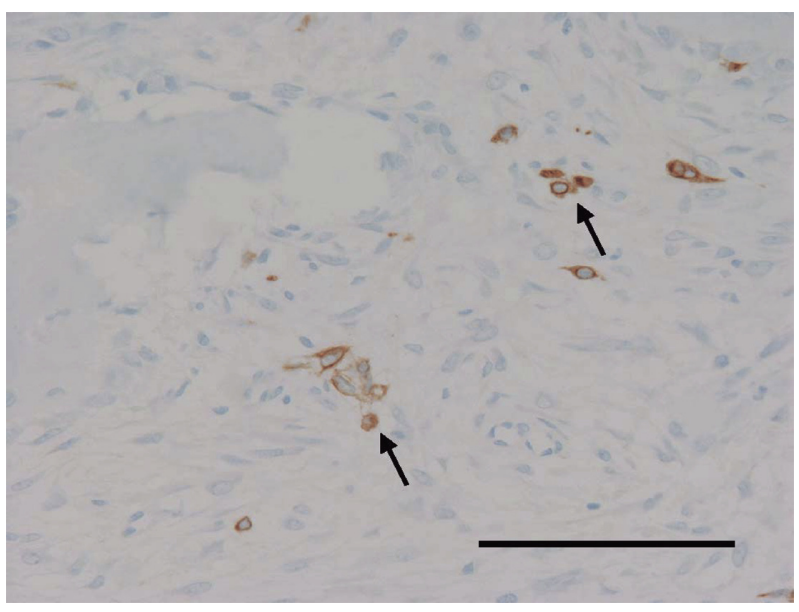

Fig. 8. Microphotograph demonstrating isolated keratin-positive epithelial cells (arrow) within the stroma. (Scale $100 \mu$ ).

\section{Discussion}

Histological features of Adamantinoma and osteofibrous dysplasia

Adamantinoma has been recognized as a primary bone tumor with epithelial characteristics and predominantly involving the tibia. Adamantinoma of the tibia can be associated with fibrous dysplasia (FD) or OFD-like areas. These areas may be predominant in some adamantinomas (Unni 1996). OFD is a rare condition, which was first described by Campanacci (1976). It most commonly affects the tibia followed by the fibula. Histologically, OFD is characterized by an osteofibrous stroma replacing normal bone, showing trabeculae which, unlike in FD, are surrounded by a regular rim of prominent cubic osteoblasts, mature lamellar bone and zonal segmentation with newly formed trabeculae mostly in the cortical region (Campanacci 1976) with radiological features similar to those of adamantinoma (Unni 1996; Kahn 2003).

\section{Correlation between Adamantinoma and osteofi- brous dysplasia I}

Several studies proposed a possible correlation between adamantinoma and OFD. Some cases of OFD have been demonstrated by immunohistochemical studies to contain cytokeratinpositive epithelial cells (Kahn 2003). In OFD, however, the epithelial-like component was much smaller than in adamantinoma and was composed of a few cells positive for cytokeratin (Benassi et al. 1994). Park et al. (1993) reviewed the clinical, radiological, and histological features of 80 cases of long bone OFD. Of the six cases of OFD immunostained with a cytokeratin antibody, two were cytokeratin positive. Certain overlapping clinical features and the morphologic similarities of many areas of OFD and adamantinoma suggest a more than coincidental association between OFD and adamantinoma (Sweet et al.1992). Evidence for the potential correlation between these two tumors is based on their similar histological features, immunohistochemical findings, the similar clonal abnormalities, overlapping skeletal distribution, and simultaneous occurrence in the tibia and fibula (Sherman et al. 2003). Results of these studies suggest that OFD and adamantinoma may be closely related lesions (Unni 1996).

\section{Correlation between Adamantinoma and osteofi- brous dysplasia II}

OFD has been considered either a regressive form of adamantinoma (Czemiak et al. 1989; Ueda et al. 1992) or as a form that has the potential to progress to adamantinoma (Bridge et al. 1994; Maki and Athanasou 2004). Maki and Athanasou (2004) analyzed the expression of several proto-oncogene products and extracellular matrix proteins by immunohistochemistry and correlated the results with histological and ultrastructural findings to investigate the correlation between OFD and adamantinoma. These findings may be consistent with the hypothesis that OFD represents a precursor lesion of adamantinoma. However, Ueda et al. (1992) also reported that no case of OFDs progressed to classic adamantinoma in their 13 cases. Sweet et al. (1992) emphasized that to date none of the 30 cases of OFD evaluated in their study has developed an adamantinoma. Park et al. (1993) also reported that adamantinoma has not developed in any of their 41 cases of OFD. Two large studies of OFD, one from Mayo Clinic and one from the American Forces Institute of Pathology, did not identify a single case which progressed from OFD to adamantinoma. The cor- 
relation between OFD and adamantinoma, if any, is therefore still totally unclear (Unni 1996).

\section{"Osteofibrous dysplasia-like" adamantinoma}

More recently, a third group of cases with clinical and radiological features similar to those of OFD has been proposed and this group of the lesion is associated with more overt strands of epithelial cells within a fibro-osseous background and have been categorized as "differentiated," "regressive," "juvenile intracortical" or "osteofibrous dysplasia-like" adamantinoma (Kahn 2003). Czerniak et al. (1989) in proposing a different correlation between OFD and adamantinoma, suggesting that adamantinoma can be divided into two distinct groups. The first group is the classic adamantinoma that involves the cortex and medulla of the tibia and usually occurs in young adults. The second group, termed differentiated adamantinoma, tends to involve children. The lesion is generally well circumscribed and cortical. These lesions are associated with uniform predominance of an OFD-like pattern, and scattered immunoreactivity of epithelial elements for cytokeratin. Sarisozen et al. (2002) reported a case of adamantinoma of the tibia in a 9-year-old boy. Histologically, the lesion was OFD with an epithelial component, called "differentiated adamantinoma." Kuruvilla and Steiner (1998) described five cases of OFD-like adamantinoma of the tibia in young patients ranging from ages 4 $1 / 2$ to 14 years. Radiologically and histologically, these cases were indistinguishable from OFD of bone, and no epithelial cells were recognized on routine staining. However, epithelial differentiation was detected in the form of scattered keratinpositive cells in all five cases, and tonofilaments in four cases. Unlike classic adamantinomas, the prognosis of OFD like adamantinoma was generally good.

\section{"Osteofibrous dysplasia-like" adamantinoma and classic adamantinoma}

There has been extensive speculation about a possible relationship between classic adamantinoma, OFD-like adamantinoma and OFD, all of which originate mainly in the tibial cortex and have close radiographic and histological similarities (Sarisozen et al. 2002). Ishida et al. (1992) studied 12 cases of OFD, two cases of OFD-like adamantinoma and five cases of classic adamantinoma clinicopathologically and immunohistochemically and reported the difference between the OFD and OFD-like adamantinoma is that the cytokeratin positive cells were scattered in the former and were both scattered and nest-like in the latter. The present case is thought to be still controversial in diagnosing OFD with a few cells positive for keratin or OFD-like adamantinoma. In World Health Organization Classification of Tumours published in 2002, Vigorita et al. (2002) clearly described that a tumor should be defined as OFD-like adamantinoma when keratin-positive epithelial cells are found. Accordingly, we diagnosed the primary lesion as OFD-like adamantinoma because of its radiological findings, predominant OFD pattern and the existence of small net-like cell groups dotted in the stroma positive for cytokeratin. On the basis of the clinical, histological, immunohistochemical and electronmicroscopical studies, a common histogenesis for classic adamantinoma, differentiated adamantinoma and osteofibrous dysplasia seems likely (Povysil et al. 2004). These studies suggest that there is an overlap or confusing of definition among OFD, OFD-like adamantinoma and classic adamantinoma and that these three lesions share the same histogenetic origin. However, surprisingly, there have been very few reports to describe subsequent progression of OFD-like adamantinoma to classic adamantinoma in spite of many conjectures that an OFD-like adamantinoma (differentiated adamantinoma) may be the precursor lesion of the classic type of adamantinoma (Kuruvilla and Steiner 1998; Sarisozen et al. 2002). Extensive literature survey reveals only two cases reported by Hazelbag et al. (1994); two patients who had had a presumed OFD-like adamantinoma, which contained few isolated keratinpositive epithelial cells within the stroma at the time of presentation, had a full-blown adamantinoma at the time of the local recurrence. In both cases the initial lesion had been thoroughly curetted so that the possibility that a classic adamanti- 
noma had been present from the inception was considered unlikely. In our case, the initial lesion was subjected to biopsy only so that the possibility that a classic adamantinoma may have been present elsewhere in the lesion cannot be entirely excluded. However, the absence of any evidence of classic adamantinoma in the original biopsy raises the possibility that, in this case, the OFDlike lesion represents a precursor lesion of classic adamantinoma.

\section{Conclusion}

OFD-like adamantinoma should be ruled out in the diagnosis of OFD. OFD-like adamantinoma may be a precursor lesion of classic adamantinoma. Therefore, the possibility of progression of OFD-like adamantinoma to a classic adamantinoma should also be considered for its treatment.

\section{References}

Benassi, M.S., Campanacci, L., Gamberi, G., Ferrari, C., Picci, P., Sangiorgi, L. \& Campanacci, M. (1994) Cytokeratin expression and distribution in adamantinoma of the long bones and osteofibrous dysplasia of tibia and fibula. An immunohistochemical study correlated to histogenesis. Histopathology, 25, 71-76.

Bridge, J.A., Dembinski, A., DeBoer, J., Travis, J. \& Neff, J.R. (1994) Clonal chromosomal abnormalities in osteofibrous dysplasia. Implications for histopathogenesis and its relationship with adamantinoma. Cancer, 73, 1746-1752.

Campanacci, M. (1976) Osteofibrous dysplasia of long bones a new clinical entity. Ital. J. Orthop. Traumatol., 2, 221-237.

Czerniak, B., Rojas-Corona, R.R. \& Dorfman, H.D. (1989) Morphologic diversity of long bone adamantinoma. The concept of differentiated (regressing) adamantinoma and its relationship to osteofibrous dysplasia. Cancer, $\mathbf{6 4}$, 2319-2334.

Hazelbag, H.M., Taminiau, A.H., Fleuren, G.J. \& Hogendoorn, P.C. (1994) Adamantinoma of the long bones. A clinicopathological study of thirty-two patients with emphasis on histological subtype, precursor lesion, and biological behavior. J. Bone Joint Surg. Am., 76, 1482-1499.

Hogendoorn, P.C.W. \& Hashimoto, H. (2002) Adamantinoma. In: Pathology and Genetics of Tumours of Soft Tissue and
Bone, edited by C.D.M., Fletcher, K.K., Unni \& M., Fredrik, LARC Press, Lyon, pp. 332-334.

Huvos, A.G. (1991) Adamantinoma of extragnathic bones. In: Bone Tumors. Diagnosis, Treatment and Prognosis, edited by A.G. Huvos, W.B.Saunders Co., Philadelephia pp. 677-693.

Ishida, T., Iijima, T., Kikuchi, F., Kitagawa, T., Tanida, T., Imamura, T. \& Machinami, R. (1992) A clinicopathological and immunohistochemical study of osteofibrous dysplasia, differentiated adamantinoma, and adamantinoma of long bones. Skeletal Radiol., 21, 493-502.

Kahn, L.B. (2003) Adamantinoma, osteofibrous dysplasia and differentiated adamantinoma. Skeletal Radiol., 32, 245-258.

Kuruvilla, G. \& Steiner, G.C. (1998) Osteofibrous dysplasialike adamantinoma of bone: a report of five cases with immunohistochemical and ultrastructural studies. Hum. Pathol., 29, 809-814.

Maki, M. \& Athanasou, N. (2004) Osteofibrous dysplasia and adamantinoma: correlation of proto-oncogene product and matrix protein expression. Hum. Pathol., 35, 69-74.

Park, Y.K., Unni, K.K., McLeod, R.A. \& Pritchard, D.J. (1993) Osteofibrous dysplasia: clinicopathologic study of 80 cases. Hum. Pathol., 24, 1339-1347.

Povysil, C., Kohout, A., Urban, K. \& Horak, M. (2004) Differentiated adamantinoma of the fibula: a rhabdoid variant. Skeletal Radiol., 33, 488-492.

Sarisozen, B., Durak, K. \& Ozturk, C. (2002) Adamantinoma of the tibia in a nine-year-old child. Acta Orthop. Belg., 68, 412-416.

Sherman, G.M., Damron, T.A. \& Yang, Y. (2003) CD99 positive adamantinoma of the ulna with ipsilateral discrete osteofibrous dysplasia. Clin. Orthop. Relat. Res., 408, 256-261.

Sweet, D.E., Vinh, T.N. \& Devaney, K. (1992) Cortical osteofibrous dysplasia of long bone and its relationship to adamantinoma. A clinicopathologic study of 30 cases. Am. J. Surg. Pathol., 16, 282-290.

Ueda, Y., Blasius, S., Edel, G., Wuisman, P., Bocker, W. \& Roessner, A. (1992) Osteofibrous dysplasia of long bones -a reactive process to adamantinomatous tissue. $J$. Cancer Res. Clin. Oncol., 118, 152-156.

Unni, K.K. (1996) "Adamantinoma" of Long Bones. In: Dahlin's Bone Tumors. General Aspects and Data on 11,087 Cases. 5th ed., Lippincott-Raven, Philadelphia, New York, pp. 333-342.

Vigorita, V., Ghelman, B. \& Hogendoorn, P.C.W. (2002) Osteofibrous dysplasia In: Pathology and Genetics of Tumours of Soft Tissue and Bone, edited by C.D.M., Fletcher, K.K., Unni \& M. Fredrik, LARC Press, Lyon, pp. 343-344. 